Chinese Journal of Organic Chemistry

\title{
四炔的芳炔环加成反应简便合成芳胺
}

\author{
吴玉芹 $a$ 于凉云 ${ }^{a}$ 张 奇 ${ }^{a}$ 李立冬*, $a, b$ \\ ( ${ }^{a}$ 盐城工学院化学与生物工程学院 盐城 224051) \\ ( ${ }^{b}$ 安徽师范大学化学与材料科学学院 芜湖 241000)
}

\begin{abstract}
摘要 芳胺作为一种合成砌块广泛存在于天然产物、医药品及分子材料中. 诸如一些亲核芳香取代、亲电硝化还原、 及过渡金属促进的氨基化合物芳基化作用等作为合成策略在 $\mathrm{C}_{\mathrm{aryl}}-\mathrm{N}$ 键形成领域被长期应用. 本工作在无金属催化条 件下通过四炔的芳炔环加成 (Hexadehydro-Diels-Alder, HDDA)反应高效合成了芳胺化合物. 该反应特征表现在无金属 催化、无添加剂、无氧化剂、无导向基团前体，反应在温和条件下高产率地得到炔官能化二环化合物并表现了优越的 区域选择性. 所合成产物的结构经各种波谱手段表征确证, 其中化合物 $\mathbf{3 h}$ 的结构还通过 X 射线单晶衍射分析确认. 关键词 芳胺; 合成; $\mathrm{C}_{\text {aryl }}-\mathrm{N}$ 偶联; HDDA 反应
\end{abstract}

\section{Simple Synthesis of Arylamines from Tetraynes via the Hexadehydro-Diels-Alder Reaction}

\author{
Wu, Yuqin ${ }^{a} \quad$ Yu, Liangyun ${ }^{a} \quad$ Zhang, $^{a}{ }^{a} \quad$ Li, Lidong*,a,b $^{*}$ \\ ( ${ }^{a}$ School of Chemical and Biological Engineering, Yancheng Institute of Technology, Yancheng 224051) \\ ( ${ }^{b}$ College of Chemistry and Materials Science, Anhui Normal University, Wuhu 241000)
}

\begin{abstract}
Arylamines are widespread in natural products, pharmaceuticals and molecular materials, and are useful synthetic building blocks. From a synthetic standpoint, the formation of $\mathrm{C}_{\text {aryl }}-\mathrm{N}$ bonds has been achieved by classical methods such as nucleophilic aromatic substitution, electrophilic nitration/reduction, or the transition-metal-mediated arylation of amides. This paper describes an efficient method for the synthesis of arylamines by the hexadehydro-Diels-Alder (HDDA) reaction of tetraynes under catalyst-free condition. This process is achieved in the absence of metal catalyst, base or oxidant, and it does not require the pre-installation of directing groups. This reaction exhibited an excellent regioselectivity, producing highly substituted fused yne-functionalized bicyclic compounds under mild conditions with good to excellent yields. All new products were fully characterized by various spectroscopic techniques and high-resolution mass spectrometry. The molecular structure of $\mathbf{3 h}$ was confirmed using single-crystal X-ray analyses.
\end{abstract}

Keywords arylamines; synthesis; $\mathrm{C}_{\text {aryl }}-\mathrm{N}$ coupling; HDDA reaction

官能化芳胺衍生物普遍存在于生物活性物质和药 物中, 被广泛应用在医药、染料、农业等领域 ${ }^{[1]} . \mathrm{C}-\mathrm{N}$ 键交叉偶联反应是形成芳胺 $\mathrm{C}-\mathrm{N}$ 键的重要策略, 通过 $\mathrm{C}-\mathrm{N}$ 偶联构建芳胺骨架成为有机合成的热点领域之一. 过渡金属催化是 $\mathrm{C}-\mathrm{N}$ 键形成的重要方法与手段, 20 世 纪 90 年代 Hartwig ${ }^{[2]}$ 和 Buchwald 等 ${ }^{[3]}$ 几乎同时独立地发 展了钯催化的 $\mathrm{C}-\mathrm{N}$ 键形成反应并被称之为 BuchwaldHartwig 交叉偶联反应. 但钯类催化剂不菲的价格、较高 的毒性、对含磷配体的依赖制约了其在工业化生产中的 应用. 20 世纪 90 年代后期起, 马大为等 ${ }^{[4]}$ 报道了铜催化
氨基酸与芳基卤的芳香化反应，推动了廉价催化剂铜在 $\mathrm{C}-\mathrm{N}$ 键形成领域的应用 ${ }^{[5]}$. 一些诸如镍 ${ }^{[6]}$ 、铁 ${ }^{[7]}$ 、铑 ${ }^{[8]}$ 等金属催化的 $\mathrm{C}-\mathrm{N}$ 键形成也得到快速发展. 近年来 $\mathrm{C}-\mathrm{N}$ 偶联形成的合成策略有了新的思路，通过芳硼酸 无金属催化直接胺化反应的研究引人注目 ${ }^{[9]}$.

Diels-Alder 反应通过[4+2]环加成对构建六元环状 体系提供了有效的策略并得以广泛应用. 近两年来, Hoye 等 ${ }^{[10]}$ 在共轭多炔环加成研究的基础上赋予 Diels-Alder 反应新的内涵, 并给这类型的反应予以新的 定义，称之为 hexadehydro-Diels-Alder (HDDA) 反应.

*E-mail: 1d-1@163.com

Received September 20, 2014; revised October 15, 2014; published online November 7, 2014.

Project supported by the National Natural Science Foundation of China (No. 21272005).

国家自然科学基金(No. 21272005)资助项目. 
HDDA 反应经历了苯炔的中间体历程, 该反应突破了以 往产生苯炔中间体 ${ }^{[11]}$ 方法的局限, 推动了芳炔化学的发 展. 同期, Lee 课题组 ${ }^{[12]}$ 在延续前期研究基础上在 HDDA 反应领域也取得了系列成果. HDDA 反应产生的 苯炔中间体因缺电子具有很强的化学活性, 易于引发分 子内或分子间亲核反应或重排反应的发生, 进而获得有 价值的芳环化合物.

我们课题组在多重烯炔 Domimo 反应 ${ }^{[13]}$ 研究基础 上发展了一种四炔芳构化卤代的 HDDA 反应 ${ }^{[14]}$. 在对 四炔环化的 HDDA 反应的苯炔中间体研究中, 我们发 现结构多样的有机胺与苯炔中间体发生亲核 $\mathrm{C}_{\text {ary }}-\mathrm{N}$ 偶 联反应，高产率地获得多官能化芳胺衍生物.

\section{1 结果与讨论}

\section{1 反应条件优化}

以底物 2,2-二(5-对氟苯基-2,4-二戊炔)丙二酸二异 丙酯和 $N, N$-二甲基苯胺的反应为模型, 对溶剂、温度及 反应时间在内的反应条件进行了优化, 结果见表 1. 首 先考察了溶剂对反应的影响(表 1, Entries 1 5). 采用极 性较低的甲苯为溶剂时, 点板发现除了主产物点之外还 伴随副产物点, 可能是异构化的产物, 此时收率为 $71 \%$ (表 1, Entry 1). 乙腈为溶剂时, 因其低沸点缘故仅得到 极低产率的产物(表 1 , Entry 3). 当用 DMF 为溶剂时, 取 得了令人满意的产率 $81 \%$ (表 1, Entry 4). 当用 DMSO 为 溶剂时, 则产率显著降低(表 1, Entry 2), 缘于 DMSO 部 分分解碎片与四炔发生了醚化反应, 该项工作我们正在 研究中.

一次偶然实验过程中混有少量水的 DMF 被作为溶 剂使用, 意外获得了更高的收率. 在对 $\mathrm{DMF} / \mathrm{H}_{2} \mathrm{O}$ 溶剂 配比中发现, 其体积比为 $10: 1$ 时反应效果最佳, 达到 $88 \%$ 的优秀产率(表 1, Entry 5). 但更多水的引入则由于 溶解性的原因, 反应效果并不令人满意. 文献查阅中发 现, Breslow 等 ${ }^{[15]}$ 在 1980 年首次发现水促进 Diels-Alder 反应进行的报道, 自此之后又有很多学者包括他本人对 这个现象进行了深入研究和阐述 ${ }^{[16]}$, 研究结果表明, 在 Diels-Alder 反应中加入水或其它极性溶剂, 可以大大促 进反应进行, 缘于水具有很高的内压力, 在憎水效应或 氢键稳定过渡态的作用下, 反应速率加快.

接着探讨了反应的最佳温度. 在 $80{ }^{\circ} \mathrm{C}$ 条件下反应 时, 仅获得 $14 \%$ 产率. 反应温度为 $90{ }^{\circ} \mathrm{C}$ 时就取得了 $71 \%$ 的产率(表 1 , Entry 7), 进一步提高温度为 $100{ }^{\circ} \mathrm{C}$ 时, 产率增高至 $88 \%$, 继续升至 $110{ }^{\circ} \mathrm{C}$ 时产率略有下降(表 1, Entry 8). 确定了最佳反应温度 $100{ }^{\circ} \mathrm{C}$ 及 $\mathrm{DMF} / \mathrm{H}_{2} \mathrm{O}$ 溶 剂, 在考察时间对反应的影响(表 1, Entry 9 12, 5)时, 对照发现 $12 \mathrm{~h}$ 为最适宜的反应时间. 最终确定该反应体
表 1 四炔无金属催化胺化反应的条件优化

Table 1 Optimization of the anilines under catalyst-free amination of tetraynes
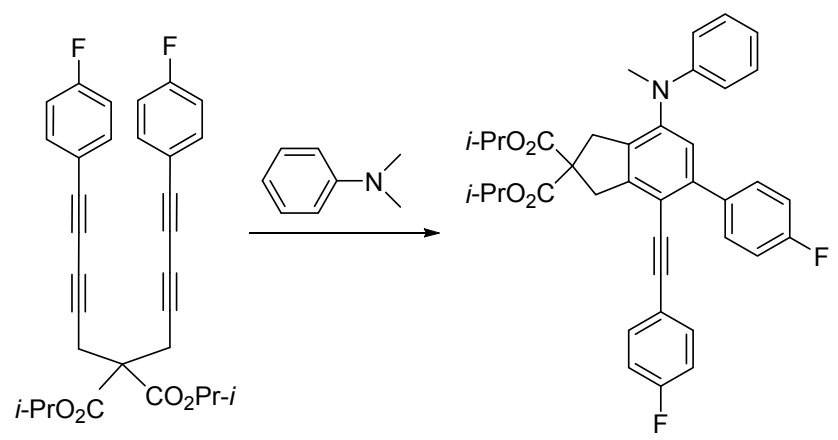

\begin{tabular}{clrrc}
\hline Entry & Solvent & $\mathrm{T}^{a} /{ }^{\circ} \mathrm{C}$ & $t / \mathrm{h}$ & Yield $^{b} / \%$ \\
\hline 1 & Toluene & 100 & 12 & 71 \\
2 & DMSO & 100 & 12 & 53 \\
3 & $\mathrm{CH}_{3} \mathrm{CN}$ & 100 & 12 & 11 \\
4 & $\mathrm{DMF}$ & 100 & 12 & 81 \\
5 & $\mathrm{DMF} / \mathrm{H}_{2} \mathrm{O}$ & 100 & 12 & 88 \\
6 & $\mathrm{DMF} / \mathrm{H}_{2} \mathrm{O}$ & 80 & 12 & 9 \\
7 & $\mathrm{DMF} / \mathrm{H}_{2} \mathrm{O}$ & 90 & 12 & 71 \\
8 & $\mathrm{DMF} / \mathrm{H}_{2} \mathrm{O}$ & 110 & 12 & 86 \\
9 & $\mathrm{DMF} / \mathrm{H}_{2} \mathrm{O}$ & 100 & 6 & 67 \\
10 & $\mathrm{DMF} / \mathrm{H}_{2} \mathrm{O}$ & 100 & 8 & 74 \\
11 & $\mathrm{DMF} / \mathrm{H}_{2} \mathrm{O}$ & 100 & 10 & 84 \\
12 & $\mathrm{DMF} / \mathrm{H}_{2} \mathrm{O}$ & 100 & 14 & 88 \\
\hline
\end{tabular}

${ }^{a}$ Oil bath temperature. ${ }^{b}$ Yield of the isolated product after flash column chromatography.

系的最优条件: 溶剂为 $\mathrm{DMF} / \mathrm{H}_{2} \mathrm{O}(V: V=10: 1)$ 的混合 溶剂, 反应温度 $100{ }^{\circ} \mathrm{C}$, 反应时间为 $12 \mathrm{~h}$.

\section{2 反应底物的适用范围}

在获得四炔胺化串联环化反应的最优反应条件后, 考察四炔底物和不同胺类在反应中的适用范围. 以碳、

氮桥连的四炔和不同类型的一级、二级、三级脂肪胺和 芳胺进行反应，结果表明，该胺化反应均能取得较高的 产率(表 2). 底物四炔的炔基末端芳环上连有氟这样的 吸电子基时产率稍高, 而连有甲氧基这样的给电子基时 产率相对要低. 四炔中桥连原子为氮原子, 反应亦能取 得满意的收率(表 2, Entries 13，18). 四炔的炔基末端连 接基团拓展为烷基时, 反应依然适用, 产率达到 $81 \%$ $83 \%$ (表 2, Entry 18).

不同类型的一级、二级、三级脂肪胺和芳胺均能同 四炔发生 HDDA 型胺化反应而获得二级、三级芳胺衍 生物. 通过选用正丁胺、炔丙胺、乙二胺、环丙胺、三 丁胺等不同脂肪胺作为胺源, 发现这些脂肪胺均能同四 炔有效反应. 该 HDDA 反应对不同结构的芳胺表现了 广适性，特意使用空间障碍较大的 2,6-二异丙基苯胺作 为底物，仍然取得了 $76 \% \sim 82 \%$ 的良好产率(表 2, Entry 18). 由此看出四炔的 HDDA 胺化反应对四炔及胺的底 物结构具有很好基团兼容性和容忍性. 
表 2 从四炔合成多官能化芳胺 ${ }^{a}$

Table 2 The synthesis of the multifunctionalized anilines from tetraynes

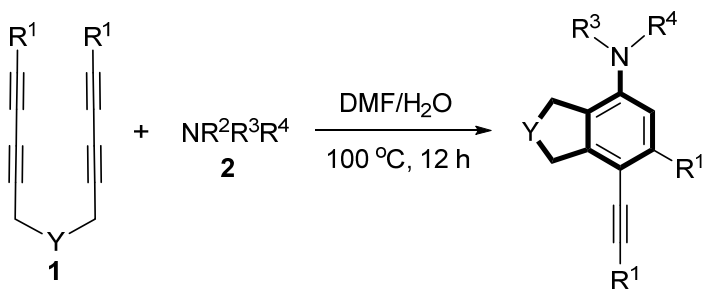

\begin{tabular}{|c|c|c|c|c|c|c|c|}
\hline Entry & Y & $\mathrm{R}^{1}$ & $\mathrm{R}^{2}$ & $\mathrm{R}^{3}$ & $\mathrm{R}^{4}$ & Product & Yield $^{b} / \%$ \\
\hline 1 & $\mathrm{C}(\mathrm{COOEt})_{2}$ & $4-\mathrm{MeC}_{6} \mathrm{H}_{4}$ & $\mathrm{H}$ & $\mathrm{H}$ & $n-\mathrm{Bu}$ & $3 \mathbf{a}$ & 72 \\
\hline 2 & $\mathrm{C}(\mathrm{COO} i-\mathrm{Pr})_{2}$ & 4- $\mathrm{FC}_{6} \mathrm{H}_{4}$ & $\mathrm{H}$ & $\mathrm{H}$ & $n-\mathrm{Bu}$ & $\mathbf{3 b}$ & 79 \\
\hline 3 & $\mathrm{C}(\mathrm{COO} i-\mathrm{Pr})_{2}$ & $\mathrm{Ph}$ & $\mathrm{H}$ & $\mathrm{H}$ & $\mathrm{HC} \equiv \mathrm{CCH}_{2}$ & $3 \mathbf{c}$ & 81 \\
\hline 4 & $\mathrm{C}(\mathrm{COO} i-\mathrm{Pr})_{2}$ & $\mathrm{Ph}$ & $\mathrm{H}$ & & Cyclopropyl & 3d & 74 \\
\hline 5 & $\mathrm{C}(\mathrm{COO} i-\mathrm{Pr})_{2}$ & $\mathrm{Ph}$ & $\mathrm{H}$ & $\mathrm{H}$ & $\mathrm{C}_{6} \mathrm{H}_{4} \mathrm{CH}_{2}$ & $3 e$ & 80 \\
\hline 6 & $\mathrm{C}(\mathrm{COOEt})_{2}$ & 4- $\mathrm{MeC}_{6} \mathrm{H}_{4}$ & $\mathrm{H}$ & $\mathrm{H}$ & $2,6-(i-\operatorname{Pr})_{2} \mathrm{C}_{6} \mathrm{H}_{3}$ & $3 f$ & 76 \\
\hline 7 & $\mathrm{C}(\mathrm{COO} i-\mathrm{Pr})_{2}$ & $4-\mathrm{FC}_{6} \mathrm{H}_{4}$ & $\mathrm{H}$ & $\mathrm{H}$ & $2,6-(i-\mathrm{Pr})_{2} \mathrm{C}_{6} \mathrm{H}_{3}$ & $3 g$ & 82 \\
\hline 8 & $\mathrm{C}(\mathrm{COOEt})_{2}$ & $\mathrm{Ph}$ & $\mathrm{H}$ & $\mathrm{H}$ & $2,6-\mathrm{Me}_{2} \mathrm{C}_{6} \mathrm{H}_{3}$ & $3 h$ & 83 \\
\hline 9 & $\mathrm{C}(\mathrm{COOMe})_{2}$ & 4- $\mathrm{MeC}_{6} \mathrm{H}_{4}$ & $\mathrm{H}$ & Et & Et & $3 \mathbf{i}$ & 82 \\
\hline 10 & $\mathrm{C}(\mathrm{COO} i-\mathrm{Pr})_{2}$ & $4-\mathrm{ClC}_{6} \mathrm{H}_{4}$ & $\mathrm{H}$ & Et & Et & $3 \mathbf{j}$ & 85 \\
\hline 11 & $\mathrm{C}(\mathrm{COO} i-\mathrm{Pr})_{2}$ & $4-\mathrm{FC}_{6} \mathrm{H}_{4}$ & $\mathrm{H}$ & & Piperidin-1-yl & $3 k$ & 81 \\
\hline 12 & $\mathrm{C}(\mathrm{COOEt})_{2}$ & 4- $\mathrm{MeC}_{6} \mathrm{H}_{4}$ & $\mathrm{H}$ & $\mathrm{Me}$ & $\mathrm{Ph}$ & 31 & 85 \\
\hline 13 & $\mathrm{TsN}$ & $4-\mathrm{FC}_{6} \mathrm{H}_{4}$ & $\mathrm{H}$ & $\mathrm{Me}$ & $\mathrm{Ph}$ & $3 m$ & 76 \\
\hline 14 & $\mathrm{C}(\mathrm{COO} i-\mathrm{Pr})_{2}$ & $4-\mathrm{FC}_{6} \mathrm{H}_{4}$ & $\mathrm{Me}$ & $\mathrm{Me}$ & $\mathrm{Ph}$ & $3 n$ & 88 \\
\hline 15 & $\mathrm{C}(\mathrm{COOMe})_{2}$ & 4- $\mathrm{ClC}_{6} \mathrm{H}_{4}$ & $\mathrm{Me}$ & $\mathrm{Me}$ & $\mathrm{Ph}$ & 30 & 82 \\
\hline 16 & $\mathrm{C}(\mathrm{COOEt})_{2}$ & 4- $\mathrm{MeOC}_{6} \mathrm{H}_{4}$ & $n-\mathrm{Bu}$ & $n-\mathrm{Bu}$ & $n-\mathrm{Bu}$ & $3 p$ & 77 \\
\hline 17 & $\mathrm{C}(\mathrm{COOEt})_{2}$ & $\mathrm{Ph}$ & $n-\mathrm{Bu}$ & $n-\mathrm{Bu}$ & $n-\mathrm{Bu}$ & $3 q$ & 85 \\
\hline 18 & TsN & $n$-Bu & $n-\mathrm{Bu}$ & $n-\mathrm{Bu}$ & $n$-Bu & $3 r$ & 78 \\
\hline
\end{tabular}

${ }^{a}$ General conditions: substrate 1 (1.0 equiv) and amine (1.1 equiv) in $7 \mathrm{~mL}$ of DMF and $0.7 \mathrm{~mL}$ of $\mathrm{H}_{2} \mathrm{O}$ at $100 \mathrm{C}$ for $12 \mathrm{~h} .{ }^{b}$ Yield of the isolated product after flash column chromatography.

\section{3 反应机理}

综合以上结果并参考相关文献 ${ }^{[10,12 \mathrm{~d}]}$, 我们认为该 反应的机理(Scheme 1)包含三步过程: (1)底物四炔在加 热状况下经历 HDDA 历程形成苯炔中间体 $\mathbf{A}$; (2)有机胺 中富电子的氮进攻缺电子苯炔形成中间体 $\mathbf{B}$; (3)中间体 B 中烯碳负离子进攻烷基 $\beta$-氢原子(底物伯胺或仲胺则 烯碳负离子进攻氮连接的氢原子), 而后此烷基经过电 子迁移最终形成烯烃离去, 同时形成胺化产物 3 .

反应中发现二甲苯胺或甲苯胺进行胺化反应均可 获得同一产物, 对于甲苯胺与四炔的反应机理可作如上 理解. 但二甲苯胺与四炔的反应机理经过氝代 DMF 为 溶剂的实验证实苯炔上的氢不是来源于溶剂, 可能来源 于二甲苯胺中的一个甲基, 但最终甲基以何种形式离 去，目前正在进一步探索之中.

\section{2 结论}

以结构多样的四炔为底物, 在无金属催化剂、无添 加剂、无氧化剂、无导向基团、无保护条件下和各式结 构不同的一级、二级、三级脂肪胺及芳胺发生 HDDA
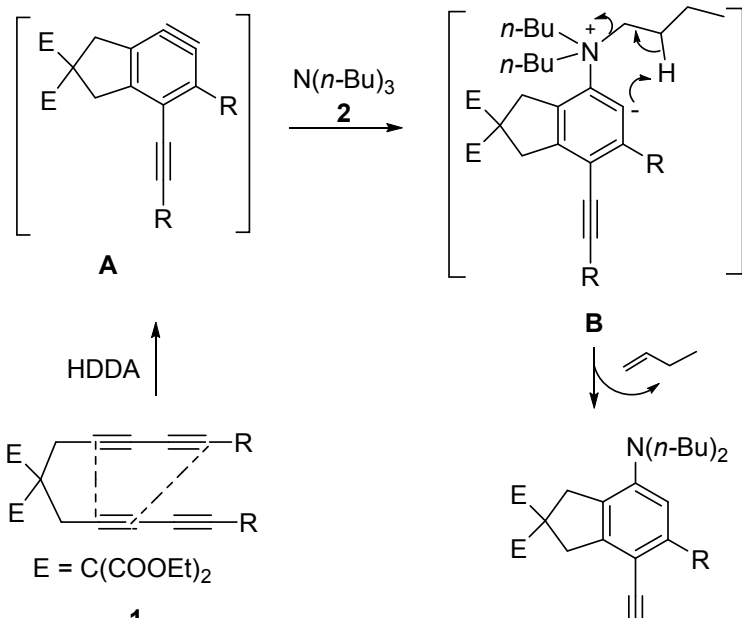

B<smiles>CCC=CCCC</smiles><smiles>[R]c1cc([N+](=O)[O-])c2c(c1C#CC)CC(F)(F)C2</smiles>

图式 1 反应的可能机理

Scheme 1 Possible reaction mechanism

型胺化反应, 得到系列二级和三级芳胺衍生物. 该反应 高效实用、操作简单、条件温和，反应表现了优秀的 
区域选择性，为芳胺类化合物的合成提供了一条实用 并具有原子经济性的途径. 所合成产物结构均经波谱手 段表征确证, 并获得化合物 $\mathbf{3 h}$ 的晶体结构(图 1), 其晶 体学数据存于英国剑桥数据中心, CCDC 号为 1024979 .

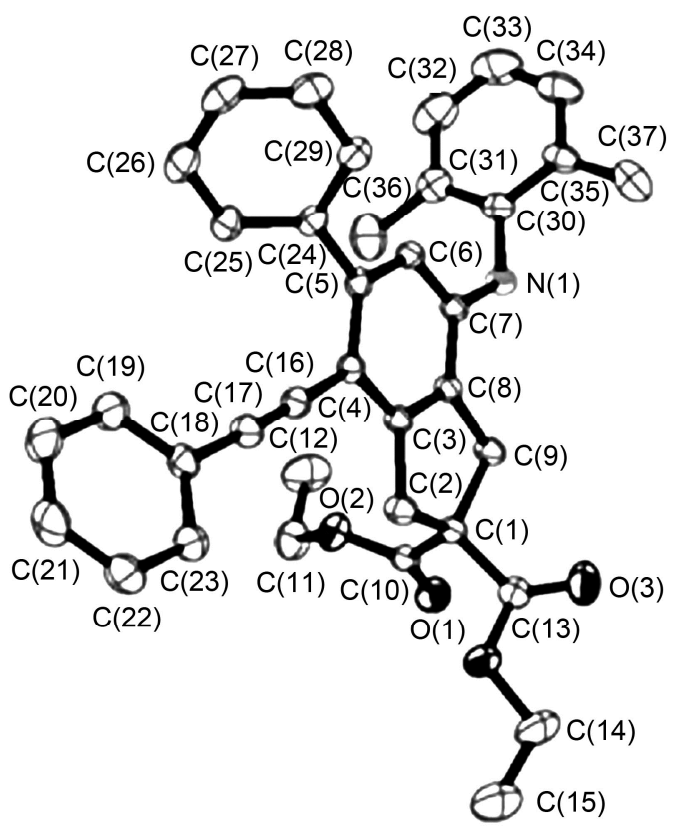

图 1 化合物 $3 \mathrm{~h}$ 的晶体结构

Figure 1 Molecular structure of compound $\mathbf{3 h}$

\section{3 实验部分}

\section{1 仪器与试剂}

仪器: 熔点用 XT-4 型双目显微熔点仪测定; 红外 光谱用 Nicole Avatar 360 傅立叶变换红外光谱仪测定 (KBr 压片); 核磁共振谱用 Bruker Avance 300 型核磁共 振谱仪测定, $\mathrm{CDCl}_{3}$ 为溶剂, $\mathrm{TMS}$ 为内标; 高分辨质谱用 Agilent 6220 质谱仪测定, APCI 电离方式. 柱层析硅胶 用 $300 \sim 400$ 目.

\section{2 实验方法}

底物合成参见文献[14]. 芳胺衍生物的一般合成步 骤: 在 $25 \mathrm{~mL}$ 的 Schlenk 反应管中加入一定质量四炔底 物(1 mmol)、胺 $(1.1 \mathrm{mmol})$, 注入 $7 \mathrm{~mL} \mathrm{DMF}$ 搅拌 $0.5 \mathrm{~h}$ 后注入 $0.7 \mathrm{~mL} \mathrm{H}_{2} \mathrm{O}$, 然后加热至 $100{ }^{\circ} \mathrm{C}$, 反应 $12 \mathrm{~h}$. 反 应结束, 冷至室温, 加入 $30 \mathrm{~mL} \mathrm{H} \mathrm{H}_{2} \mathrm{O}$, 用 $30 \mathrm{~mL}$ 乙酸乙 酯萃取有机层 3 次. 有机相经无水 $\mathrm{MgSO}_{4}$ 干燥后蒸除低 沸物, 所得粗产物经硅胶柱层析[洗脱剂: $V$ (石油醚)： $V($ 乙酸乙酯 $)=20: 1$ ]淋洗, 得到最终的胺化产物.

5-对甲苯基-4-对甲苯乙炔基-7-正丁氨基- $1 H$-狮2,2(3H)-乙二酸二乙酯(3a): 白色固体, 产率 72\%. m.p. $131 \sim 132{ }^{\circ} \mathrm{C} ;{ }^{1} \mathrm{H}$ NMR $\left(300 \mathrm{MHz}, \mathrm{CDCl}_{3}\right) \delta: 7.56$ (d, $J=$ $8.1 \mathrm{~Hz}, 2 \mathrm{H}), 7.26 \sim 7.18(\mathrm{~m}, 4 \mathrm{H}), 7.07$ (d, $J=7.8 \mathrm{~Hz}, 2 \mathrm{H})$, $6.48(\mathrm{~s}, 1 \mathrm{H}), 4.28 \sim 4.21(\mathrm{~m}, 4 \mathrm{H}), 3.81(\mathrm{~s}, 2 \mathrm{H}), 3.55(\mathrm{~s}$, $1 \mathrm{H}), 3.43(\mathrm{~s}, 2 \mathrm{H}), 3.18(\mathrm{t}, J=6.9 \mathrm{~Hz}, 2 \mathrm{H}), 2.41(\mathrm{~s}, 3 \mathrm{H})$, $2.33(\mathrm{~s}, 3 \mathrm{H}), 1.66 \sim 1.59(\mathrm{~m}, 2 \mathrm{H}), 1.47 \sim 1.39(\mathrm{~m}, 2 \mathrm{H})$, 1.29 (t, $J=6.9 \mathrm{~Hz}, 6 \mathrm{H}), 0.96(\mathrm{t}, J=7.2 \mathrm{~Hz}, 3 \mathrm{H}) ;{ }^{13} \mathrm{C} \mathrm{NMR}$ $\left(75 \mathrm{MHz}, \mathrm{CDCl}_{3}\right) \delta: 171.8,144.6,144.0,143.9,138.5$, $137.3,136.8,130.9,129.2,128.9,128.5,122.4,121.4$, 109.5, 106.0, 93.4, 87.8, 61.9, 59.4, 43.3, 41.4, 37.8, 31.6, 21.5, 21.3, 20.3, 14.1, 14.0; IR (KBr) v: 3387, 2976, 2951, $1726,1601,1510,1364,1273,1190,1069,1013,812,741$ $\mathrm{cm}^{-1}$; HRMS (APCI) calcd for $\mathrm{C}_{35} \mathrm{H}_{40} \mathrm{NO}_{4}[\mathrm{M}+\mathrm{H}]^{+}$ 538.2952, found 538.2947.

5-对氟苯基-4-对氟苯乙炔基-7-正丁氨基- $1 H$-茚2,2(3H)-乙二酸二异丙酯 $(\mathbf{3 b})$ : 白色固体，产率 $79 \%$. m.p. 202 203 ${ }^{\circ} \mathrm{C} ;{ }^{1} \mathrm{H}$ NMR (300 MHz, $\left.\mathrm{CDCl}_{3}\right) \delta: 7.57$ $(\mathrm{d}, J=6.0 \mathrm{~Hz}, 2 \mathrm{H}), 7.28 \sim 7.21(\mathrm{~m}, 2 \mathrm{H}), 7.11(\mathrm{t}, J=7.2$ $\mathrm{Hz}, 2 \mathrm{H}), 6.97$ (t, J=7.2 Hz, 2H), $6.43(\mathrm{~s}, 1 \mathrm{H}), 5.12 \sim 5.04$ $(\mathrm{m}, 2 \mathrm{H}), 3.75(\mathrm{~s}, 2 \mathrm{H}), 3.59(\mathrm{~s}, 1 \mathrm{H}), 3.89(\mathrm{~s}, 2 \mathrm{H}), 3.18(\mathrm{t}$, $J=6.0 \mathrm{~Hz}, 2 \mathrm{H}), 1.66 \sim 1.59(\mathrm{~m}, 2 \mathrm{H}), 1.47 \sim 1.40(\mathrm{~m}, 2 \mathrm{H})$, $1.26(\mathrm{~d}, J=6.3 \mathrm{~Hz}, 12 \mathrm{H}), 0.96(\mathrm{t}, J=7.2 \mathrm{~Hz}, 3 \mathrm{H}) ;{ }^{13} \mathrm{C}$ NMR $\left(75 \mathrm{MHz}, \mathrm{CDCl}_{3}\right) \delta: 171.2,163.8\left(J_{\mathrm{C}-\mathrm{F}}=258.4 \mathrm{~Hz}\right)$, $163.6\left(J_{\mathrm{C}-\mathrm{F}}=247.8 \mathrm{~Hz}\right), 144.2,137.4,132.7,132.6,131.0$, $130.9,122.8,115.6,115.3,114.7,114.5,109.3,92.2,87.8$, $69.4,59.3,43.3,41.2,37.7,31.6,21.6,20.3,13.9$; IR (KBr) v: 3389, 2984, 2936, 1732, 1560, 1506, 1366, 1273, 1252, 1227, 1182, 1107, 1065, 878, 831, 750, $694 \mathrm{~cm}^{-1}$; HRMS (APCI) calcd for $\mathrm{C}_{35} \mathrm{H}_{38} \mathrm{~F}_{2} \mathrm{NO}_{4}[\mathrm{M}+\mathrm{H}]^{+}$574.2763, found 574.2756 .

5-苯基-4-苯乙炔基-7-炔丙氨基- $1 H$-狮-2,2(3H)-乙 二酸二异丙酯 $(3 \mathrm{c})$ : 白色固体，产率 $81 \%$. m.p. 124 $125{ }^{\circ} \mathrm{C} ;{ }^{1} \mathrm{H}$ NMR $\left(300 \mathrm{MHz}, \mathrm{CDCl}_{3}\right) \delta: 7.65$ (d, $J=6.9$ $\mathrm{Hz}, 2 \mathrm{H}), 7.46 \sim 7.36(\mathrm{~m}, 3 \mathrm{H}), 7.29 \sim 7.24(\mathrm{~m}, 5 \mathrm{H}), 6.59(\mathrm{~s}$, $1 \mathrm{H}), 5.13 \sim 5.04(\mathrm{~m}, 2 \mathrm{H}), 4.01(\mathrm{~s}, 2 \mathrm{H}), 3.89(\mathrm{~s}, 1 \mathrm{H}), 3.79$ $(\mathrm{s}, 2 \mathrm{H}), 3.44(\mathrm{~s}, 2 \mathrm{H}), 2.27(\mathrm{~s}, 1 \mathrm{H}), 1.26(\mathrm{~d}, J=6.0 \mathrm{~Hz}$, $12 \mathrm{H}) ;{ }^{13} \mathrm{C}$ NMR $\left(75 \mathrm{MHz}, \mathrm{CDCl}_{3}\right) \delta: 171.2,144.8,144.4$, $142.6,141.1,131.1,129.5,128.2,127.9,127.5,127.4$, $124.2,123.8,110.4,107.5,93.7,88.2,80.5,72.0,69.5$, 59.5, 41.3, 47.8, 33.3, 21.6; IR (KBr) v: 3374, 3319, 3051, 2984, 1724, 1595, 1491, 1373, 1346, 1281, 1260, 1196, 1144, 1105, 1069, 835, 748, $683 \mathrm{~cm}^{-1}$; HRMS (APCI) calcd for $\mathrm{C}_{34} \mathrm{H}_{34} \mathrm{NO}_{4}[\mathrm{M}+\mathrm{H}]^{+}$520.2482, found 520.2480.

5-苯基-4-苯乙炔基-7-环丙氨基- $1 H$-狮-2,2(3H)-乙 二酸二异丙酯(3d): 白色固体，产率 74\%. m.p. 119 $120{ }^{\circ} \mathrm{C} ;{ }^{1} \mathrm{H}$ NMR $\left(300 \mathrm{MHz}, \mathrm{CDCl}_{3}\right) \delta: 7.68$ (d, $J=6.9$ $\mathrm{Hz}, 2 \mathrm{H}), 7.47 \sim 7.37(\mathrm{~m}, 3 \mathrm{H}), 7.32 \sim 7.23(\mathrm{~m}, 5 \mathrm{H}), 6.91(\mathrm{~s}$, $1 \mathrm{H}), 5.12 \sim 5.04(\mathrm{~m}, 2 \mathrm{H}), 3.79(\mathrm{~s}, 2 \mathrm{H}), 3.37(\mathrm{~s}, 2 \mathrm{H}), 2.50$ 
(s, 1H), $1.26(\mathrm{~d}, J=6.3 \mathrm{~Hz}, 12 \mathrm{H}), 1.16(\mathrm{~d}, J=6.3 \mathrm{~Hz}, 1 \mathrm{H})$, $0.75(\mathrm{~d}, J=5.7 \mathrm{~Hz}, 2 \mathrm{H}), 0.57$ (s, 2H); ${ }^{13} \mathrm{C}$ NMR $(75 \mathrm{MHz}$, $\left.\mathrm{CDCl}_{3}\right) \delta: 171.2,144.7,144.4,144.1,141.4,131.0,129.5$, $128.2,127.8,127.4,127.2,124.4,122.8,110.9,106.6$, 93.4, 88.4, 69.4, 59.4, 41.3, 37.7, 25.0, 21.6, 7.7; IR (KBr) $v: 3381,3057,2980,1721,1595,1491,1452,1358,1285$, 1196, 1103, 1067, 910, 816, 758, 690, $525 \mathrm{~cm}^{-1}$; HRMS (APCI) calcd for $\mathrm{C}_{34} \mathrm{H}_{36} \mathrm{NO}_{4}[\mathrm{M}+\mathrm{H}]^{+} 522.2639$, found 522.2635 .

5-苯基-4-苯乙炔基-7-芐氨基- $1 H$-狮-2,2(3H)-乙二 酸二异丙酯(3e): 白色固体, 产率 80\%. m.p. 132 $133{ }^{\circ} \mathrm{C} ;{ }^{1} \mathrm{H}$ NMR $\left(300 \mathrm{MHz}, \mathrm{CDCl}_{3}\right) \delta: 7.57$ (d, $J=6.3$ $\mathrm{Hz}, 2 \mathrm{H}), 7.41 \sim 7.34(\mathrm{~m}, 7 \mathrm{H}), 7.33 \sim 7.27(\mathrm{~m}, 7 \mathrm{H}), 6.58(\mathrm{~s}$, 1H), $5.13 \sim 5.04(\mathrm{~m}, 2 \mathrm{H}), 4.41(\mathrm{~s}, 2 \mathrm{H}), 3.80(\mathrm{~s}, 2 \mathrm{H}), 3.46$ (s, 2H), $1.26(\mathrm{~d}, J=6.3 \mathrm{~Hz}, 12 \mathrm{H}) ;{ }^{13} \mathrm{C}$ NMR $(75 \mathrm{MHz}$, $\left.\mathrm{CDCl}_{3}\right) \delta: 171.2,144.9,144.2,143.8,141.2,138.6,131.0$, $129.4,128.8,128.2,127.8,127.8,127.6,127.4,127.3$, $124.4,123.1,109.9,106.5,93.5,88.4,69.4,59.4,48.0$, 41.4, 37.9, 21.6; IR (KBr) v: 3387, 2980, 1726, 1593, 1493, 1373, 1277, 1103, 1055, 910, 752, $700 \mathrm{~cm}^{-1}$; HRMS (APCI) calcd for $\mathrm{C}_{38} \mathrm{H}_{38} \mathrm{NO}_{4}[\mathrm{M}+\mathrm{H}]^{+}$572.2795, found 572.2797 .

5-对甲苯基-4-(对甲苯乙炔基)-7-[(2,6-二异丙苯基) 氨基]- $1 H$-狮-2,2(3H)-乙二酸二乙酯(3f): 白色固体, 产 率 76\%. m.p. 162 163 ${ }^{\circ} \mathrm{C} ;{ }^{1} \mathrm{H}$ NMR $\left(300 \mathrm{MHz}, \mathrm{CDCl}_{3}\right)$ $\delta: 7.35(\mathrm{~d}, J=7.5 \mathrm{~Hz}, 2 \mathrm{H}), 7.25 \sim 7.17(\mathrm{~m}, 5 \mathrm{H}), 7.14 \sim$ $7.06(\mathrm{~m}, 4 \mathrm{H}), 6.02(\mathrm{~s}, 1 \mathrm{H}), 5.00(\mathrm{~s}, 1 \mathrm{H}), 4.32 \sim 4.25(\mathrm{~m}$, $4 \mathrm{H}), 3.87(\mathrm{~s}, 2 \mathrm{H}), 3.61(\mathrm{~s}, 2 \mathrm{H}), 3.21 \sim 3.12(\mathrm{~m}, 2 \mathrm{H}), 2.34$ (s, 3H), $2.32(\mathrm{~s}, 3 \mathrm{H}), 1.3(\mathrm{t}, J=7.2 \mathrm{~Hz}, 6 \mathrm{H}), 1.18 \sim 1.13$ $(\mathrm{m}, 12 \mathrm{H}) ;{ }^{13} \mathrm{C}$ NMR $\left(75 \mathrm{MHz}, \mathrm{CDCl}_{3}\right) \delta: 171.8,147.4$, $144.5,144.3,143.7,138.1,137.4,136.8,134.3,131.0$, $129.1,128.9,128.4,127.7,123.9,122.2,121.3,111.1$, $107.1,110.9,93.7,87.5,62.0,59.7,41.5,37.9,28.4,24.6$, 23.3, 14.1; IR (KBr) v: 3373, 3022, 2959, 1722, 1599, 1493, 1362, 1259, 1180, 1072, 864, 816, $606 \mathrm{~cm}^{-1}$; HRMS (APCI) calcd for $\mathrm{C}_{43} \mathrm{H}_{48} \mathrm{NO}_{4}[\mathrm{M}+\mathrm{H}]^{+} 642.3578$, found 642.3570 .

5-对氟苯基-4-(对氟苯乙炔基)-7-[(2,6-二异丙苯基) 氨基]- $1 H$-䒢-2,2 $(3 H)$-乙二酸二异丙酯 $(\mathbf{3 g})$ : 白色固体, 产率 82\%. m.p. $148 \sim 149{ }^{\circ} \mathrm{C}$; ${ }^{1} \mathrm{H}$ NMR $(300 \mathrm{MHz}$, $\left.\mathrm{CDCl}_{3}\right) \delta: 7.41 \sim 7.37(\mathrm{~m}, 2 \mathrm{H}), 7.29 \sim 7.19(\mathrm{~m}, 5 \mathrm{H})$, $7.03 \sim 6.93(\mathrm{~m}, 4 \mathrm{H}), 6.00(\mathrm{~s}, 1 \mathrm{H}, \mathrm{Ar}-\mathrm{H}), 5.17 \sim 5.09(\mathrm{~m}$, $2 \mathrm{H}), 5.04(\mathrm{~s}, 1 \mathrm{H}), 3.82\left(\mathrm{~s}, 2 \mathrm{H}, \mathrm{CCH}_{2}\right), 3.59\left(\mathrm{~s}, 2 \mathrm{H}, \mathrm{CCH}_{2}\right)$, $3.19 \sim 3.11(\mathrm{~m}, 2 \mathrm{H}), 1.29(\mathrm{~d}, J=5.7 \mathrm{~Hz}, 12 \mathrm{H}), 1.14(\mathrm{dd}$, $J=6.9,6.9 \mathrm{~Hz}, 12 \mathrm{H}) ;{ }^{13} \mathrm{C}$ NMR $\left(75 \mathrm{MHz}, \mathrm{CDCl}_{3}\right) \delta$ :
$171.1,163.8\left(J_{\mathrm{C}-\mathrm{F}}=246.1 \mathrm{~Hz}\right), 163.7\left(J_{\mathrm{C}-\mathrm{F}}=248.8 \mathrm{~Hz}\right)$, $147.4,144.5,143.9,134.0,132.8,132.7,130.9,127.9$, $124.0,122.6,115.5,115.3,114.6,114.3,110.7,106.7$, $92.5,87.5,69.5,59.7,41.3,37.8,28.4,24.5,23.2,21.6$; IR (KBr) $v: 3389,2970,1709,1603,1578,1466,1354,1157$, $1105,835,752,687 \mathrm{~cm}^{-1}$; HRMS (APCI) calcd for $\mathrm{C}_{43} \mathrm{H}_{46} \mathrm{~F}_{2} \mathrm{NO}_{4} 678.3389[\mathrm{M}+\mathrm{H}]^{+}$, found 678.3383

5-苯基-4-苯乙炔基-7-[(2,6-二甲苯基)氨基]- $1 H$-狮2,2(3H) -乙二酸二乙酯 $(\mathbf{3 h})$ : 白色固体, 产率 $83 \%$. m.p. $197 \sim 198{ }^{\circ} \mathrm{C} ;{ }^{1} \mathrm{H}$ NMR $\left(300 \mathrm{MHz}, \mathrm{CDCl}_{3}\right) \delta: 7.49(\mathrm{~d}, J=$ $7.2 \mathrm{~Hz}, 2 \mathrm{H}), 7.35 \sim 7.23(\mathrm{~m}, 8 \mathrm{H}), 7.08(\mathrm{~d}, J=6.3 \mathrm{~Hz}, 3 \mathrm{H})$, $6.07(\mathrm{~s}, 1 \mathrm{H}), 5.08(\mathrm{~s}, 1 \mathrm{H}), 4.30 \sim 4.27(\mathrm{~m}, 4 \mathrm{H}), 3.88(\mathrm{~s}$, $2 \mathrm{H}), 3.60(\mathrm{~s}, 2 \mathrm{H}), 2.23(\mathrm{~s}, 6 \mathrm{H}), 1.31(\mathrm{t}, J=6.9 \mathrm{~Hz}, 12 \mathrm{H})$; ${ }^{13} \mathrm{C}$ NMR (75 MHz, $\mathrm{CDCl}_{3}$ ) $\delta: 171.7,144.7,144.6,142.2$, $140.9,137.2,136.0,131.1,129.4,128.7,128.2,127.7$, $127.5,127.2,126.4,124.3,123.2,111.2,107.2,93.7,88.2$, 62.0, 59.7, 41.4, 37.9, 18.4, 14.2; IR (KBr) v: 3372, 3030, 2980, 1711, 1605, 1491, 1362, 1304, 1248, 1188, 1070, $866,756,690 \mathrm{~cm}^{-1}$; HRMS (APCI) calcd for $\mathrm{C}_{37} \mathrm{H}_{36} \mathrm{NO}_{4}$ $558.2639[\mathrm{M}+\mathrm{H}]^{+}$, found 558.2633.

5-对甲苯基-4-对甲苯乙炔基-7-[( $N, N$-二乙基)氨 基]- $1 H$-狮-2,2(3H)-乙二酸二甲酯(3i): 黄色液体, 产率 $82 \% .{ }^{1} \mathrm{H}$ NMR $\left(300 \mathrm{MHz}, \mathrm{CDCl}_{3}\right) \delta: 7.54(\mathrm{~d}, J=7.8 \mathrm{~Hz}$, 2H), $7.26(\mathrm{~s}, 4 \mathrm{H}), 7.10(\mathrm{~s}, 4 \mathrm{H}), 6.77(\mathrm{~s}, 1 \mathrm{H}), 3.85(\mathrm{~s}, 2 \mathrm{H})$, $3.79(\mathrm{~s}, 6 \mathrm{H}), 3.62(\mathrm{~s}, 2 \mathrm{H}), 3.18(\mathrm{~d}, J=6.0 \mathrm{~Hz}, 4 \mathrm{H}), 2.41(\mathrm{~s}$, $3 \mathrm{H}), 2.34(\mathrm{~s}, 3 \mathrm{H}), 1.30 \sim 1.09(\mathrm{~m}, 6 \mathrm{H}) ;{ }^{13} \mathrm{C}$ NMR $(75 \mathrm{MHz}$, $\left.\mathrm{CDCl}_{3}\right) \delta: 172.3,147.1,144.7,143.2,138.1,137.7,136.8$, $131.1,130.3,129.2,128.9,128.5,121.0,118.8,109.9$, 94.4, 87.3, 59.7, 53.1, 45.5, 41.3, 40.5, 21.5, 21.3, 12.8; IR (KBr) v: 3036, 2948, 1737, 1593, 1512, 1457, 1365, 1269, $1071,814,647 \mathrm{~cm}^{-1}$; HRMS (APCI) calcd for $\mathrm{C}_{33} \mathrm{H}_{36} \mathrm{NO}_{4}$ $[\mathrm{M}+\mathrm{H}]^{+}$510.2639, found 510.2633.

5-对氯苯基-4-对氯苯乙炔基-7-[( N,N-二乙基)氨 基]- $1 H$-狮-2,2(3H)-乙二酸二异丙酯 $(\mathbf{3 j})$ ：白色固体，产 率 85\%. m.p. $150 \sim 151{ }^{\circ} \mathrm{C} ;{ }^{1} \mathrm{H}$ NMR $\left(300 \mathrm{MHz}, \mathrm{CDCl}_{3}\right)$ $\delta: 7.59$ (d, $J=6.9 \mathrm{~Hz}, 2 \mathrm{H}), 7.44$ (s, 2H), 7.29 (s, 4H), 6.74 (s, 1H), 5.13 5.03 (m, 2H), $3.81(\mathrm{~s}, 2 \mathrm{H}), 3.61(\mathrm{~s}, 2 \mathrm{H})$, $3.26(\mathrm{~s}, 4 \mathrm{H}), 1.31(\mathrm{~s}, 12 \mathrm{H}), 1.29 \sim 1.12(\mathrm{~m}, 6 \mathrm{H}) ;{ }^{13} \mathrm{C} \mathrm{NMR}$ $\left(75 \mathrm{MHz}, \mathrm{CDCl}_{3}\right) \delta: 171.5,147.3,145.5,142.2,139.4$, $133.5,133.2,132.2,130.8,130.7,128.6,128.0,122.4$, 118.4, 109.2, 93.4, 88.6, 69.0, 59.7, 45.6, 40.8, 40.3, 21.7, 12.5; IR (KBr) v: 3056, 2967, 2872, 1731, 1587, 1521, 1489, 1376, 1251, 1196, 1097, 827, 724, $652 \mathrm{~cm}^{-1}$; HRMS (APCI) calcd for $\mathrm{C}_{35} \mathrm{H}_{38} \mathrm{Cl}_{2} \mathrm{NO}_{4}[\mathrm{M}+\mathrm{H}]^{+}$606.2172, found 606.2169 . 
5-对氟苯基-4-对氟苯乙炔基-7-戊环氨基- $1 H$-狮2,2(3H)-乙二酸二异丙酯 $(3 \mathbf{k})$ : 白色固体, 产率 $81 \%$. m.p. $175 \sim 176{ }^{\circ} \mathrm{C} ;{ }^{1} \mathrm{H}$ NMR $\left(300 \mathrm{MHz}, \mathrm{CDCl}_{3}\right) \delta: 7.55(\mathrm{t}$, $J=8.4 \mathrm{~Hz}, 2 \mathrm{H}), 7.27$ (d, $J=7.2 \mathrm{~Hz}, 2 \mathrm{H}), 7.07$ (d, $J=9.0$ $\mathrm{Hz}, 2 \mathrm{H}), 6.95$ (d, $J=8.4 \mathrm{~Hz}, 2 \mathrm{H}), 6.73(\mathrm{~s}, 1 \mathrm{H}), 5.11 \sim 5.03$ $(\mathrm{m}, 2 \mathrm{H}), 3.72(\mathrm{~s}, 2 \mathrm{H}), 3.56(\mathrm{~s}, 2 \mathrm{H}), 3.01(\mathrm{~s}, 4 \mathrm{H}), 1.71(\mathrm{~s}$, 4H), 1.58 (s, 2H), $1.26(\mathrm{~d}, J=6.0 \mathrm{~Hz}, 12 \mathrm{H}) ;{ }^{13} \mathrm{C} \mathrm{NMR}(75$ $\left.\mathrm{MHz}, \mathrm{CDCl}_{3}\right) \delta: 171.2,163.9\left(J_{\mathrm{C}-\mathrm{F}}=246.5 \mathrm{~Hz}\right), 163.8$ $\left(J_{\mathrm{C}-\mathrm{F}}=247.3 \mathrm{~Hz}\right), 150.1,144.8,142.9,133.0,132.9,131.0$, $130.9,117.3,115.7,115.4,114.8,114.5,93.3,87.3,69.3$, 60.0, 51.9, 40.9, 39.8, 26.3, 24.3, 21.6; IR (KBr) v: 3027, 2956, 1726, 1602, 1506, 1365, 1256, 1168, 1057, 837, 741 $\mathrm{cm}^{-1}$; HRMS (APCI) calcd for $\mathrm{C}_{71} \mathrm{H}_{77} \mathrm{~F}_{2} \mathrm{~N}_{2} \mathrm{O}_{8}[\mathrm{M}+\mathrm{H}]^{+}$ 586.2763, found 586.2757.

5-对甲苯基-4-对甲苯乙炔基-7-[( $N$-甲基- $N$-苯基)氨 基]-1H-狮-2,2(3H)-乙二酸二乙酯(3I): 白色固体, 产率 85\%. m.p. 91 92 ${ }^{\circ} \mathrm{C} ;{ }^{1} \mathrm{H}$ NMR (300 MHz, $\left.\mathrm{CDCl}_{3}\right) \delta$ : $7.56(\mathrm{~d}, J=8.1 \mathrm{~Hz}, 2 \mathrm{H}), 7.30 \sim 7.20(\mathrm{~m}, 6 \mathrm{H}), 7.10(\mathrm{t}, J=$ $7.5 \mathrm{~Hz}, 2 \mathrm{H}), 6.86 \sim 6.77(\mathrm{~m}, 3 \mathrm{H}), 4.23 \sim 4.16(\mathrm{~m}, 4 \mathrm{H}), 3.81$ $(\mathrm{s}, 2 \mathrm{H}), 3.33(\mathrm{~s}, 3 \mathrm{H}), 3.29(\mathrm{~s}, 2 \mathrm{H}), 2.41(\mathrm{~s}, 3 \mathrm{H}), 2.35$ (s, $3 \mathrm{H}), 1.24(\mathrm{t}, J=6.9 \mathrm{~Hz}, 6 \mathrm{H}) ;{ }^{13} \mathrm{C} \mathrm{NMR}\left(75 \mathrm{MHz}, \mathrm{CDCl}_{3}\right)$ : $\delta 171.6,148.3,145.5,144.2,144.2,138.2,137.4,137.2$, $134.6,131.4,131.3,129.1,129.1,128.9,128.7,124.7$, 120.7, 119.2, 116.3, 95.7, 86.8, 61.8, 59.5, 41.2, 40.0, 39.6, 21.6, 21.3, 14.1; IR (KBr) v: 3022, 2980, 1728, 1587, 1497, 1476, 1364, 1279, 1248, 1180, 1157, 1107, 1070, $860,814,750,692 \mathrm{~cm}^{-1}$; HRMS (APCI) calcd for $\mathrm{C}_{38} \mathrm{H}_{38} \mathrm{NO}_{4}[\mathrm{M}+\mathrm{H}]^{+} \quad$ 572.2795, found 572.2789.

6-对氟苯基-7-对氟苯乙炔基- $N$-甲基- $N$-苯基-2-对 甲苯磺酰基异吲哚啉-4-胺(3m): 白色固体, 产率 76\%. m.p. $192 \sim 193{ }^{\circ} \mathrm{C} ;{ }^{1} \mathrm{H}$ NMR $\left(300 \mathrm{MHz}, \mathrm{CDCl}_{3}\right) \delta: 7.61$ $(\mathrm{d}, J=8.4 \mathrm{~Hz}, 2 \mathrm{H}), 7.58 \sim 7.54(\mathrm{~m}, 2 \mathrm{H}), 7.31 \sim 7.22(\mathrm{~m}$, $6 \mathrm{H}), 7.12$ (t, $J=8.7 \mathrm{~Hz}, 2 \mathrm{H}), 7.02$ (t, $J=8.4 \mathrm{~Hz}, 4 \mathrm{H}), 6.82$ $(\mathrm{d}, J=8.4 \mathrm{~Hz}, 2 \mathrm{H}), 4.71(\mathrm{~s}, 2 \mathrm{H}), 4.07(\mathrm{~s}, 2 \mathrm{H}), 2.31(\mathrm{~s}, 3 \mathrm{H})$, $2.42(\mathrm{~s}, 3 \mathrm{H}) ;{ }^{13} \mathrm{C} \mathrm{NMR}\left(75 \mathrm{MHz}, \mathrm{CDCl}_{3}\right) \delta: 163.6\left(J_{\mathrm{C}-\mathrm{F}}=\right.$ $245.6 \mathrm{~Hz}), 147.8,144.1,143.9,143.6,141.4,133.5,133.2$, $133.1,130.9,130.8,129.8,129.4,128.8,127.7,122.0$, $119.8,115.8,115.1,114.9,110.4,94.9,85.5,54.4,53.8$, $40.8,21.5$; IR (KBr) v: 3061, 2953, 1605, 1508, 1495, 1373, 1342, 1217, 1167, 1098, 1055, 839, $667 \mathrm{~cm}^{-1}$; HRMS (APCI) calcd for $\mathrm{C}_{36} \mathrm{H}_{29} \mathrm{~F}_{2} \mathrm{~N}_{2} \mathrm{O}_{2} \mathrm{~S} \quad[\mathrm{M}+\mathrm{H}]^{+}$ 591.1912, found 591.1909.

5-对氟苯基-4-对氟苯乙炔基-7-[( $N$-甲基- $N$-苯基)氨 基]-1 $H$-狮-2,2(3H)-乙二酸二异丙酯(3n): 白色固体, 产 率 88\%. m.p. $128 \sim 129{ }^{\circ} \mathrm{C} ;{ }^{1} \mathrm{H}$ NMR (300 $\mathrm{MHz}, \mathrm{CDCl}_{3}$ ) $\delta: 7.61 \sim 7.59(\mathrm{~m}, 2 \mathrm{H}), 7.34 \sim 7.20(\mathrm{~m}, 4 \mathrm{H}), 7.11(\mathrm{t}, J=9.0$ $\mathrm{Hz}, 2 \mathrm{H}), 7.00(\mathrm{t}, J=8.4 \mathrm{~Hz}, 3 \mathrm{H}), 6.86 \sim 6.78(\mathrm{~m}, 3 \mathrm{H})$, $5.07 \sim 4.99(\mathrm{~m}, 2 \mathrm{H}), 3.74(\mathrm{~s}, 2 \mathrm{H}), 3.32(\mathrm{~s}, 3 \mathrm{H}), 3.24(\mathrm{~s}$, $3 \mathrm{H}), 1.20(\mathrm{dd}, J=6.3,6.3 \mathrm{~Hz}, 12 \mathrm{H}) ;{ }^{13} \mathrm{C} \mathrm{NMR}(75 \mathrm{MHz}$, $\left.\mathrm{CDCl}_{3}\right) \delta: 171.1,164.0\left(J_{\mathrm{C}-\mathrm{F}}=247.1 \mathrm{~Hz}\right), 148.3,145.7$, $144.6,143.4,135.0,133.2,133.1,131.0,130.9,129.2$, $124.4,119.5,116.6,115.8,115.5,115.0,114.7,94.5,86.8$, 69.3, 59.5, 41.0, 40.0, 39.6, 21.6; IR (KBr) v: 2984, 2936, 2897, 1732, 1599, 1506, 1366, 1273, 1227, 1182, 1107, $1065,878,831,750,694 \mathrm{~cm}^{-1}$; HRMS (APCI) calcd for $\mathrm{C}_{38} \mathrm{H}_{36} \mathrm{~F}_{2} \mathrm{NO}_{4}[\mathrm{M}+\mathrm{H}]^{+}$608.2608, found 608.2602.

5-对氯苯基-4-对氯苯乙炔基-7-[( $N$-甲基- $N$-苯基)氨 基]-1H-狮-2,2(3H)-乙二酸二甲酯(3o)：白色固体，产率 82\%. m.p. 111 $112{ }^{\circ} \mathrm{C}$; ${ }^{1} \mathrm{H}$ NMR (300 MHz, $\left.\mathrm{CDCl}_{3}\right) \delta$ : 7.55 (d, $J=9.0 \mathrm{~Hz}, 2 \mathrm{H}), 7.38$ (d, $J=8.7 \mathrm{~Hz}, 2 \mathrm{H}), 7.28 \sim$ $7.22(\mathrm{~m}, 7 \mathrm{H}), 7.04(\mathrm{~s}, 1 \mathrm{H}), 6.79$ (d, J=7.8 Hz, 2H), 3.78 $(\mathrm{s}, 2 \mathrm{H}), 3.73(\mathrm{~s}, 6 \mathrm{H}), 3.34(\mathrm{~s}, 3 \mathrm{H}), 3.24(\mathrm{~s}, 2 \mathrm{H}) ;{ }^{13} \mathrm{C} \mathrm{NMR}$ $\left(75 \mathrm{MHz}, \mathrm{CDCl}_{3}\right) \delta: 171.9,148.1,145.6,144.8,143.3$, $138.6,134.5,134.2,133.6,132.6,132.5,130.6,129.2$, $128.7,128.1,123.9,121.9,120.0,117.2,113.5,113.0$, 112.6, 94.6, 88.0, 59.4, 53.1, 41.2, 40.2, 39.8; IR (KBr) v: 3028, 2933, 1735, 1593, 1492, 1361, 1250, 1013, 829, 752 $\mathrm{cm}^{-1}$; HRMS (APCI) calcd for $\mathrm{C}_{34} \mathrm{H}_{28} \mathrm{Cl}_{2} \mathrm{NO}_{4}[\mathrm{M}+\mathrm{H}]^{+}$ 584.1390, found 584.1389.

5-对甲氧苯基-4-对甲氧苯乙炔基-7-[( $N, N$-二丁基) 氨基]- $1 H$-狮-2,2(3H)-乙二酸二乙酯(3p): 黄色液体, 产 率 77\%. ${ }^{1} \mathrm{H}$ NMR $\left(300 \mathrm{MHz}, \mathrm{CDCl}_{3}\right) \delta: 7.58$ (d, $J=6.3$ $\mathrm{Hz}, 2 \mathrm{H}), 7.26$ (d, $J=8.1 \mathrm{~Hz}, 2 \mathrm{H}), 6.97 \sim 6.95$ (d, $J=6.6$ $\mathrm{Hz}, 2 \mathrm{H}), 6.79$ (d, $J=8.1 \mathrm{~Hz}, 2 \mathrm{H}), 6.75(\mathrm{~s}, 1 \mathrm{H}), 4.21$ (d, $J=$ $7.2 \mathrm{~Hz}, 4 \mathrm{H}), 3.85$ (s, 3H), 3.78 (s, 3H), 3.76 (s, 2H), 3.58 (s, 2H), $3.10(\mathrm{~d}, J=6.6 \mathrm{~Hz}, 4 \mathrm{H}), 2.41(\mathrm{~d}, J=5.1 \mathrm{~Hz}, 4 \mathrm{H})$, $1.47 \sim 1.36(\mathrm{~m}, 4 \mathrm{H}), 1.27(\mathrm{t}, J=7.2 \mathrm{~Hz}, 4 \mathrm{H}), 0.90(\mathrm{t}, J=$ $6.9 \mathrm{~Hz}, 6 \mathrm{H}) ;{ }^{13} \mathrm{C} \mathrm{NMR}\left(75 \mathrm{MHz}, \mathrm{CDCl}_{3}\right) \delta: 171.4,159.1$, $158.9,147.4,144.9,142.8,133.6,132.8,132.3,130.3$, $118.7,116.3,113.8,113.0,110.1,94.3,86.7,61.9,59.9$, 55.1, 52.0, 41.1, 40.4, 29.8, 20.3, 14.2, 14.1; IR (KBr) $v$ : 3042, 2967, 1728, 16047, 1509, 1466, 1283, 1251, 1172, 1068, 831, $672 \mathrm{~cm}^{-1}$; HRMS (APCI) calcd for $\mathrm{C}_{39} \mathrm{H}_{48} \mathrm{NO}_{6}$ $[\mathrm{M}+\mathrm{H}]^{+}$625.3555, found 626.3473 .

5-苯基-4-苯乙炔基-7-[( N,N-二丁基)氨基 $]-1 H$-茚2,2(3H)-乙二酸二乙酯 $(\mathbf{3 q})$ : 黄色液体，产率 $85 \% ;{ }^{1} \mathrm{H}$ NMR (300 MHz, $\left.\mathrm{CDCl}_{3}\right) \delta: 7.64(\mathrm{~d}, J=7.2 \mathrm{~Hz}, 2 \mathrm{H})$, $7.46 \sim 7.36(\mathrm{~m}, 4 \mathrm{H}), 7.31 \sim 7.24(\mathrm{~m}, 4 \mathrm{H}), 6.50(\mathrm{~s}, 1 \mathrm{H})$, $4.29 \sim 4.22(\mathrm{~m}, 4 \mathrm{H}), 3.82(\mathrm{~s}, 2 \mathrm{H}), 3.44(\mathrm{~s}, 2 \mathrm{H}), 3.22 \sim 3.18$ $(\mathrm{m}, 4 \mathrm{H}), 1.67 \sim 1.59(\mathrm{~m}, 4 \mathrm{H}), 1.29(\mathrm{t}, J=6.9 \mathrm{~Hz}, 6 \mathrm{H}), 0.96$ 
$(\mathrm{t}, J=7.2 \mathrm{~Hz}, 6 \mathrm{H}) ;{ }^{13} \mathrm{C} \mathrm{NMR}\left(75 \mathrm{MHz}, \mathrm{CDCl}_{3}\right) \delta: 171.7$, $147.7,145.1,143.5,141.0,131.1,130.7,129.4,128.1$, $127.8,127.5,127.2,124.1,118.9,109.6,94.3,88.1,61.8$, 59.4, 51.8, 41.4, 40.5, 29.6, 21.0, 20.3, 14.0; IR (KBr) v: 3048, 2961, 1732, 1587, 1465, 1361, 1243, 1179, 1071, $863,752 \mathrm{~cm}^{-1}$; HRMS (APCI) calcd for $\mathrm{C}_{39} \mathrm{H}_{48} \mathrm{NO}_{6}[\mathrm{M}+$ $\mathrm{H}]^{+}$626.3476, found 626.3473 .

$N, N, 6$-三丁基-7-(1-己炔基)-2-对甲苯磺酰基异吲哚 啉-4-胺(3r)：黄色液体，产率 78\%; ${ }^{1} \mathrm{H}$ NMR $(300 \mathrm{MHz}$, $\left.\mathrm{CDCl}_{3}\right) \delta: 7.75(\mathrm{~d}, J=8.1 \mathrm{~Hz}, 2 \mathrm{H}), 7.28(\mathrm{~d}, J=7.8 \mathrm{~Hz}$, $2 \mathrm{H}), 6.50(\mathrm{~s}, 1 \mathrm{H}), 4.58(\mathrm{~s}, 2 \mathrm{H}), 4.52(\mathrm{~s}, 2 \mathrm{H}), 2.97(\mathrm{t}, J=6.6$ $\mathrm{Hz}, 2 \mathrm{H}), 2.63$ (t, $J=6.6 \mathrm{~Hz}, 2 \mathrm{H}), 2.43$ (d, $J=6.0 \mathrm{~Hz}, 2 \mathrm{H})$, $2.39(\mathrm{~s}, 3 \mathrm{H}), 1.58 \sim 1.42(\mathrm{~m}, 6 \mathrm{H}), 1.35 \sim 1.19(\mathrm{~m}, 10 \mathrm{H})$, $0.97 \sim 0.83(\mathrm{~m}, 12 \mathrm{H}) ;{ }^{13} \mathrm{C} \mathrm{NMR}\left(75 \mathrm{MHz}, \mathrm{CDCl}_{3}\right) \delta$ : $145.3,145.2,143.5,140.4,133.7,129.8,127.6,124.7$, $118.2,109.9,96.5,76.3,54.4,54.3,52.0,34.3,33.0,31.0$, $29.6,22.6,22.0,21.5,20.2,19.3,14.0,14.0,13.7$; IR (KBr) v: 2957, 2930, 1601, 1489, 1458, 1352, 1165, 1098, 1063, 814, $669 \mathrm{~cm}^{-1}$; HRMS (APCI) calcd for $\mathrm{C}_{33} \mathrm{H}_{49^{-}}$ $\mathrm{N}_{2} \mathrm{O}_{2} \mathrm{~S}[\mathrm{M}+\mathrm{H}]^{+}$537.3487, found 537.3492.

辅助材料(Supporting Information) 产物的核磁氢谱、 碳谱谱图及化合物 $\mathbf{3 h}$ 的晶体数据. 这些材料可以免费 从本刊网站(http://sioc-journal.cn/)上下载.

\section{References}

[1] (a) Hartwig, J. F. Nature 2008, 455, 314.

(b) Hartwig, J. F.; Stanley, L. M. Acc. Chem. Res. 2010, 43, 1461.

(c) Chen, G. F., Ma, S. Angew. Chem., Int. Ed. 2010, 49, 8306.

[2] (a) Paul, F.; Patt, J.; Hartwig, J. F. J. Am. Chem. Soc. 1994, 116, 5969.

(b) Louie, J.; Hartwig, J. F. Tetrahedron Lett. 1995, 36, 3609.

(c) Stambuli, J. P.; Kuwano, P.; Hartwig, J. F. Angew. Chem. Int. Ed. 2002, 41, 4746.

[3] (a) Guram, A. S.; Buchwald, S. L. J. Am. Chem. Soc. 1994, 116, 7901 .

(b) Rennels, R. A.; Buchwald, S. L. Angew. Chem., Int. Ed. 1995, 34, 1348 .

(c) Wolf, J. P.; Buchwald, S. L. Angew. Chem., Int. Ed. 1999, 38, 2413.

[4] (a) Ma, D.; Zhang, Y.; Yao, J.; Wu, S.; Tao, F. J. Am. Chem. Soc. 1998, 120, 12459

(b) Ma, D.; Xia, D. Org. Lett. 2001, 3, 2583.

(c) Ma, D.; Cai, Q.; Zhang, H. Org. Lett. 2003, 5, 2453.

(d) Zhang, H.; Cai, Q.; Ma, D. J. Org. Chem. 2005, 70, 5164.

[5] Zhang, J.; Yin, H.; Han, S. Chin. J. Org. Chem. 2012, 32, 1429 (in Chinese).

(张敬先，殷慧清，韩世清，有机化学, 2012, 32, 1429.)
[6] (a) Wolfe, J. P.; Buchwald, S. L. J. Am. Chem. Soc. 1997, 119, 6054 .

(b) Ge, S.; Green, R. A.; Hartwig, J. F. J. Am. Chem. Soc. 2014, $136,1617$.

[7] Guo, D. L.; Huang, H.; Xu, J. Y. Org. Lett. 2008, 10, 4513.

[8] Louillat, M. L.; Biafora, A.; Legros, F.; Patureau, F. W. Angew. Chem., Int. Ed. 2014, 53, 3505.

[9] (a) Coeffard, V.; Moreau, X.; Thomassigny, C.; Greck, C. Angew. Chem., Int. Ed. 2013, 52, 5684.

(b) Xiao, Q.; Tian, L.; Tan, R.; Xia, Y.; Qiu, D.; Zhang, Y.; Wang, J. Org. Lett. 2012, 14, 4230.

(c) Mlynarski, S. N.; Karns, A. S.; Morken, J. P. J. Am. Chem. Soc. 2012, 134, 16449 .

(d) Zhu, C.; Li, G.; Ess, D. H.; Falck, J. R.; Kürti, L. J. Am. Chem. Soc. 2012, 134, 18253.

[10] (a) Hoye, T. R.; Baire, B.; Niu, D.; Willoughby, P. H.; Woods, B. P. Nature 2012, 490, 208.

(b) Niu, D.; Hoye, T. R. Nat. Chem. 2013, 6, 34.

(c) Baire, B.; Niu, D.; Willoughby, P. H.; Woods, B. P.; Hoye, T. R. Nat. Protoc. 2013, 8, 501.

(d) Niu, D.; Willoughby, P. H.; Woods, B. P.; Baire, B.; Hoye, T. R. Nature 2013, 501, 531.

(e) Niu, D.; Wang, T.; Woods, B. P.; Hoye, T. R. Org. Lett. 2014, 16, 254.

[11] (a) Tadross, P. M.; Stoltz, B. M. Chem. Rev. 2012, 112, 3550.

(b) Gampe, C. M.; Carreira, E. M. Angew. Chem., Int. Ed. 2012, 51, 3766 .

(c) Hoffmann, R. W.; Suzuki, K. Angew. Chem., Int. Ed. 2013, 52, 2655.

(d) Holden, C.; Greaney, M. F. Angew. Chem., Int. Ed. 2014, 53, 5746.

[12] (a) Yun, S. Y.; Wang, K.-P.; Lee, N.-K.; Mamidipalli, P.; Lee, D. J. Am. Chem. Soc. 2013, 135, 4668.

(b) Wang, K.-P.; Yun, S. Y.; Mamidipalli, P.; Lee, D. Chem. Sci. 2013, 4, 3205.

(c) Karmakar, R.; Mamidipalli, P.; Yun, S. Y.; Lee, D. Org. Lett. 2013, 15, 1938.

(d) Karmakar, R.; Yun, S. Y.; Wang, K.-P.; Lee, D. Org. Lett. 2014, 16,6 .

(e) Mamidipalli, P.; Yun, S. Y.; Wang, K.-P.; Zhou, T.; Xia, Y.; Lee, D. Chem. Sci. 2014, 5, 2362.

[13] (a) Hu, Y. M.; Yu, C. L.; Ren, D.; Hu, Q.; Zhang, L. D.; Cheng, D. Angew. Chem., Int. Ed. 2009, 48, 5448.

(b) Hu, Y. M.; Ouyang, Y.; Qu, Y.; Hu, Q.; Yao, H. Chem. Commun. 2009, 4575.

(c) Hu, Y.; Yao, H.; Sun, Y.; Wan, J.; Lin, X.; Zhu, T. Chem.-Eur. J. 2010, 16, 7635.

(d) Zhao, Q. S.; Hu, Q.; Wen, L.; Wu, M.; Hu, Y. M. Adv. Synth. Catal. 2012, 354, 2113.

(e) Li, Li.; Hu, Q.; Zhou, P.; Xie, H.; Zhang, X.; Hu, Y. Org. Biomol. Chem. 2014, 12, 5356.

[14] Zhang, H.; Hu, Q.; Li, L.; Hu, Y.; Zhou, P.; Zhang, X.; Xie, H.; Yin, F.; Hu, Y.; Wang, S. Chem. Commun. 2014, 50, 3335.

[15] Rideout, D. C.; Breslow, R. J. Am. Chem. Soc. 1980, 102, 7816.

[16] (a) Breslow, R.; Rizzo, C. J. J. Am. Chem. Soc. 1991, 113, 4340. (b) Hake, R.; McLendon, G.; Corin, A.; Holzschu, D. J. Am. Chem. Soc. 1992, 114, 5442 . 